\title{
Effect of SDBS on the drag reduction characteristics of polyacrylamide in a rotating disk apparatus
}

\author{
Hayder A. Abdul Bari ${ }^{1,2}$, Zainab Yousif ${ }^{1 *}$, Zulkefli Bin Yaacob ${ }^{1}$, Edward Oluwasogaakindoyo ${ }^{1}$ \\ ${ }^{1}$ Faculty of Chemical and Natural Resources Eng., University Malaysia Pahang, Kuantan, Malaysia \\ ${ }^{2}$ Center of Excel.for Adv. Res. in Fluid Flow (CARIFF), University Malaysia Pahang, Kuantan, Malaysia \\ *Corresponding author E-mail: zyou1973@gmail.com
}

Copyright () 2015 Hayder A. Abdul Bari et al. This is an open access article distributed under the Creative Commons Attribution License, which permits unrestricted use, distribution, and reproduction in any medium, provided the original work is properly cited.

\begin{abstract}
This paper focuses on the determination of the interaction between polymer (Polyacrylamide (PAM)) and surfactant (Sodium dod benzene sulfonate (SDBS)) as a drag reducer using Rotating Disk apparatus (RDA) at various concentrations $(500,700,1000,1500$ and 2000) ppm individually and in the combined form under turbulent conditions at different rotation speed up to $3000 \mathrm{rpm}$, as well as studying their mechanisms as a drag reducer. The results show that the maximum percent drag reduction increases to $(40,41,43,45$ and 48)\% by using the combined additives of surfactant and polymer at the above concentrations respectively, with slower degradation and display drag reduction for a larger range of Reynolds numbers. The nano and micro particles formed from the combined PAA and SDBSA was studied using cryo-transmission electron microscopy (cryo-TEM) techniques. The images show the surrounding of polymer chain to the surfactant micelle to form an aggregate structure. A hexagonal crystalline form was suggested to describe the shape of the aggregate structure.
\end{abstract}

Keywords: Drag Reduction; Complexes; Polymer; Surfactant; TEM.

\section{Introduction}

Polymeric drag reducing agents are considered as successful and commercially feasible additives for enhancing the flow in strategic pipelines. It is widely believed that drag reduction in the presence of polymer is caused by the suppression of both eddy growth and eddy formation. Hassan [1] have shown that in the presence of polymer, the turbulent energy production is decreased.

The mechanism of its drag reduction depends mainly on the Physiochemical properties as well as surface tension. The lowering of the surface tension of solvent by addition of surfactant stops when surfactant molecules begin to form micelles in the bulk solution. The concentration at which micelles start to form is called critical micelle concentration (CMC). At concentrations sufficiently higher than the CMC surfactant molecules form warm like structures in turbulent flow. Worm-like micellar structures are crucial for drag reduction in turbulent flow. Small-angle neutron scattering of surfactant induced drag reducing solution reveals anisotropic conditions where rodlike micelles align and orient themselves parallel to the direction of flow above the critical shear stress. Below the critical shear stress, we observe freely rotating micelles with no ordered orientation [2] (Bewersdorff 1986). The The DR of combination for two-phase liquids, dilute polymer and surfactant solutions — have been studied previously by numerous researchers [3-10]

The presence of polymer with surfactant micelle-like aggregates begin to form at a critical aggregation concentration ( $\mathrm{cmc}$ ). Thus $\mathrm{cmc}$ is an analogue of $\mathrm{cmc}$ in solutions of surfactants with added polymeric component. A characteristic feature of this parameter is that it is always lower than the cmc of the corresponding surfactant. Binding of surfactant to the polymer, which may depend on several parameters, such as charge density, surfactant structure, and ionic strength, can lead to the formation of characteristic micro- and macrostructure, [6] proposed a mechanism for surfactant-polymer drag reduction, he observed 
that an interaction between polymer and surfactant causes an aggregation.These structures responsible for drag reduction.

In this work, an enclosed Rotating Disk apparatus (RDA) was used to study the drag reduction effectives of ionic polymer (polyacriamaide) and anionic surfactant (Sodium dodbenzensulfonate (SDBS)) individually and in combined form. As well as investigation of the effect of concentration, rotation speed on drag reduction efficiency, its mechanical degradation and stability with time at turbulent condition.

\section{Material and methods}

Polyacrylamide and Sodium dodbenzensulfonate are used as drag reducer. Different concentration of them were prepared in distilled water. The concentrations tested for polymer and surfactant are 500, 1000, 1500 and $2000 \mathrm{ppm}$. All samples were stored in a container at room temperature of one day to get dissolved properly. The Brookfield viscometer in the concentric cylinder mode is used to determine the rheological properties of the test samples.

Drag reduction measurements were implemented using rotating disc apparatus (RDA). The RDA consists of a simple disk, made of aluminum with a diameter of $18 \mathrm{~cm}$ and a height of $1.9 \mathrm{~cm}$, so that drag reduction efficiency can be evaluated. The disk could be rotated at a radius of $\mathrm{a}=8 \mathrm{~cm}$ and a thickness of $\mathrm{b}=0.3 \mathrm{~cm}$ (see Fig. 1). Heidolph Company, ModelRZR 2102 has manufactured the rotor system, enclosed by constant speed. With this rotor system, the rotational speed of the disk could be controlled from 0 up to a maximum of $3000 \mathrm{rpm}$. The used volume of solution necessary to fill the entire container is about $1200 \mathrm{~cm} 3$.

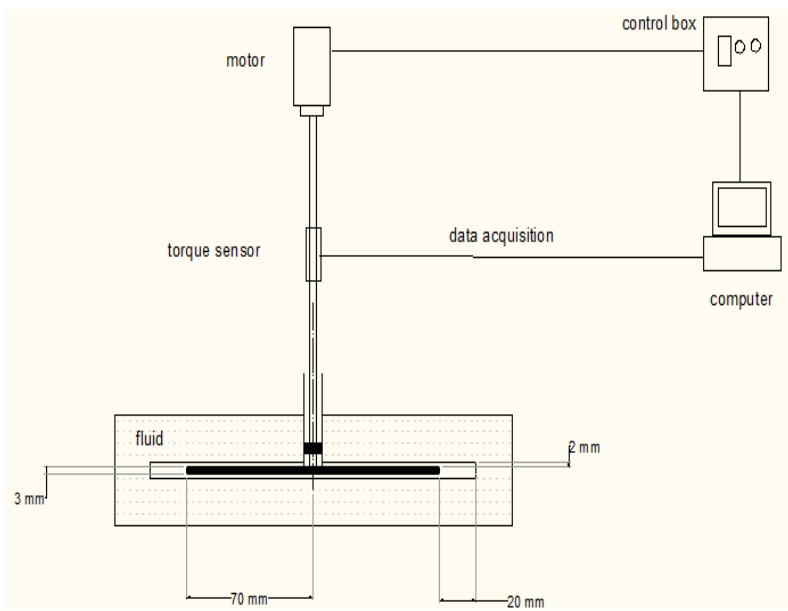

Fig. 1: Schematic of (RDA) Rotating Disk Apparatus

Computer control unit combining high-speed data sampling with controlled disk and rotational speed are integrated into the RDA system to measure fluid friction from laminar to turbulent flow accurately. The rotating disk apparatus could calculate both skin and form friction in which the internal flow shows the frictional drag reduction while external flow shows total drag that includes both flow over flat plates (skin friction) and flow around submerged particles(form friction). Nevertheless, frictional part is only related to drag reduction phenomenon.

The drag reduction efficiency of any drag reduction agent can be represented as given below:

$\% \mathrm{Dr}=\frac{\mathrm{Tw}-\mathrm{Tc}}{\mathrm{Tw}} \times 100$

Where: Tw and Tc are the torque's output for the (polymer and surfactant combines) measured on both sides of the disk, rotating at the same rpm in the water with and without additives with surfactant and polymers, respectively.

The Reynolds number of the disk can be determining by:

$\mathrm{NRe}=\frac{\rho \mathrm{R}^{2} \omega}{\mu}$

Where: $\mu$ : is the fluid viscosity; $\rho$ : is fluid densityR and $\omega$ are the radius of the disk and the angular velocity, respectively.

Turbulence is yield by using the rotary disk apparatuses, for NRe $>3 * 105$. 


\section{Results and discussion}

As we mentioned before, both of polymer and surfactant are the most common materials that used as a drag reducer, in spite of the difference in their mechanism. For polymers the drag reduction depends mainly on stretching and aggregation of polymer molecules that occurs due to shear stress. As shear stress increases i.e. turbulent intensity increases, this induces stretching of polymer chain, which increases effective viscosity in the buffer layer of turbulent flow by increasing elongation viscosity [11-12] leading to decay of turbulent eddies and increasing in percent drag reduction as shown in Fig.2. These values of drag reduction percentages increases with increasing rotation speed till they reach maximum values of $(28,30,33$, 35, and $36) \%$ for polymer concentration $(500,700,1000,1500$ and 2000)ppm respectively.But these molecules undergo mechanical degradation under high shear condition leading to lose their drag reduction capability.

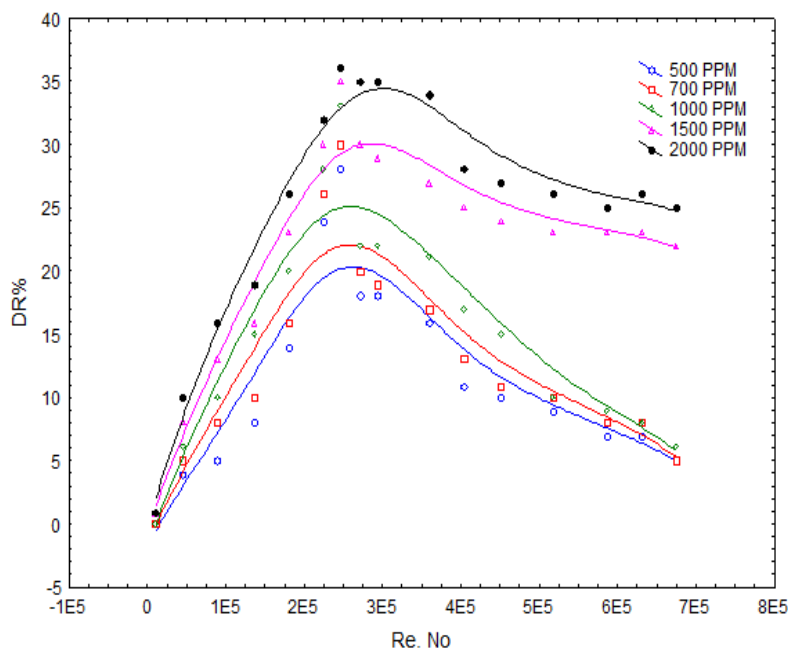

Fig. 2: The Percentage of Drag Reduction with Reynolds Number at Different Polymer Concentrations

For more declaration on the mechanical degradation.Fig. 3 shows the dependence of drag reduction percentage with time for different polymer concentrations at certain rotation speed of $3000 \mathrm{rpm}$. In which, the polymer is more shear stable drag reduction agent at higher concentration (1500 and 2000)ppm more than that at lower concentration $(500,700$ and 1000)ppm. This degradation causes the polymer chains to be broken into smaller chains which are not effective as drag reducing agents.

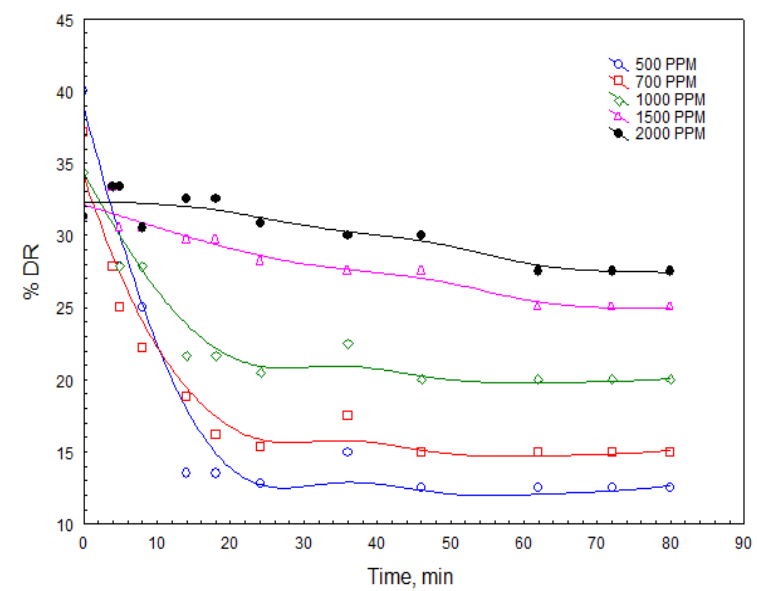

Fig. 3: The Effect of Time on Percent Drag Reduction for Different Polymer Concentration at 3000 Rpm.

The drag percent drag reduction also depends on the concentration of polymer. Generally, it is seen to increase with increasing concentration as shown in Figs.2-3. This behavior presumably due to increasing in viscosity, i.e. increasing in number of molecular, which causes the decay of more turbulent, eddies. The same trend of percent drag reduction dependence on shear stress and concentration can be seen with surfactant additives but with different mechanism that depends mainly on the viscoelasticity behavior of the surfactant solution and its rheological properties. The elasticity of the fluid helps to suppress turbulent coherent structures such as eddies and vortices. Fig. 4 shows the relation of drag reduction with Reynolds number (shear stress) at different surfactant concentrations. As the velocity increase, the drag reduction increases too 
and reach maximum values of $(30,32,34,37$ and 40) \% for $(500,700,1000,1500$ and 2000) ppm concentration. The reduction occurs when the rode like micelles grow rapidly, as the concentration exceed critical micelles concentration, forming a three-dimensional threadlike micelles. This threadlike micelles usually exhibit shear thinning behavior. As a result of the non-uniform stress distribution during experiments, these micelles are oriented such that their long axis is parallel to the flow direction in shear.

Although, the mechanical stability of the anionic surfactant [13], but they seem to be degraded as shown in Fig.5, this degradation occurs as a result of break-up of the micelle microstructure due to high shear stresses that exceed critical stress. The decreasing in drag reduction can be also explained if we know that for anionic surfactant the spherical micelles usually aggregate into cylindrical micelles, which formed a network of interlaced rod-like elements and the aggregation forces of the surfactant molecules are overcome by the shear stress so the aggregates are broken down to small particles, which have little drag reduction ability.

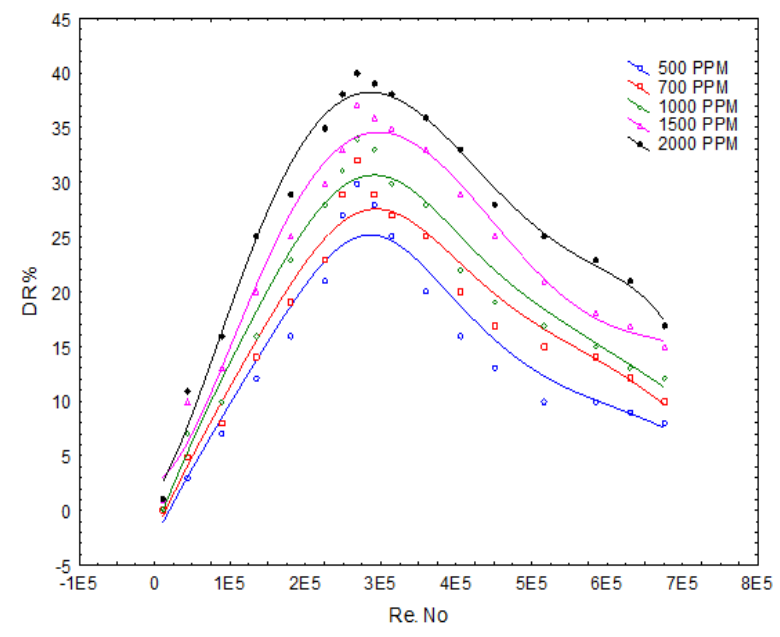

Fig. 4: The Percentage of Drag Reduction with Reynolds Number at Different Surfactant

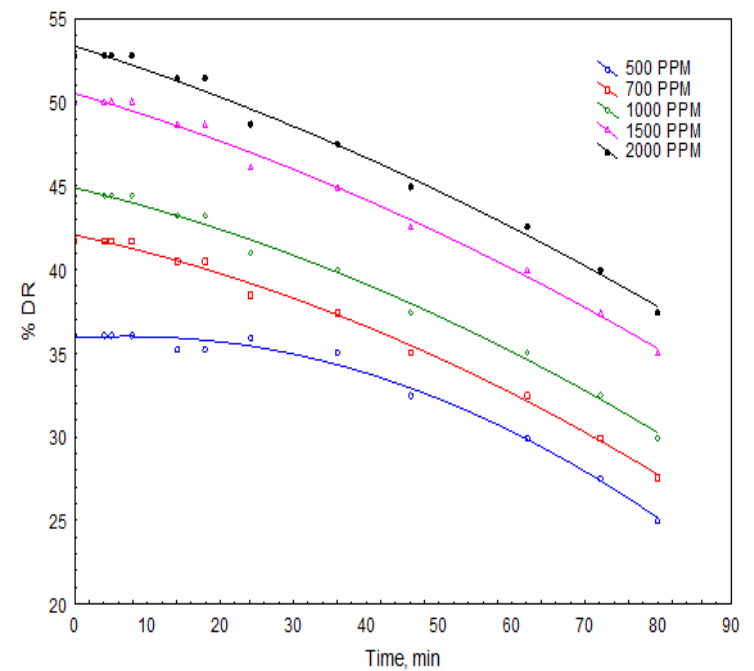

Fig. 5: The Effect of Time on Percent Drag Reduction for Different Surfactant Concentration at 3000 Rpm

Also, as the surfactant concentration increases the drag reduction increases too (figs.4 - 5) due to increasing with the length of rod-like micelles and increasing with an aggregation number of spherical micelles, similar results shown by other workers [14-16].In order to study the effect of surfactant addition on the drag reduction behavior of Polyacrylamide, other experiments were done using the tow additives with equal concentrations under different shear stress. The results from these experiments are given in figs. (6-7). the maximum drag reduction gained are (40, 41, 43, 45 and 48) \% for concentrations (500, 700, 1000,1500 and 2000) ppm, respectively. These percents are higher than that of polymer or surfactant individually with shifting in Reynolds number, at which maximum reduction occurs, from 270000 to 315000 . This shifting induced to the interaction between surfactant and polymer chain, in which that polymer film is formed around surfactant micelles forming aggregate structure. This structure has drastic effect on the solution rheology. They take an ordered orientation that minimizes resistance to flow, with increasing flow they elongated and suppress the turbulent eddies. Hence the drag reduction increases with shear stress. 


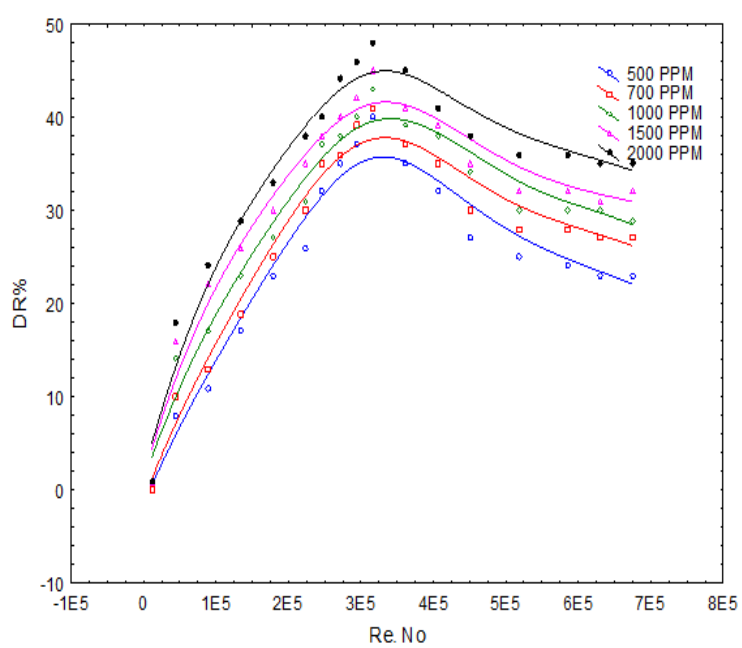

Fig. 6: The Percentage of Drag Reduction with Reynolds Number at Different Polymer-Surfactant Concentrations

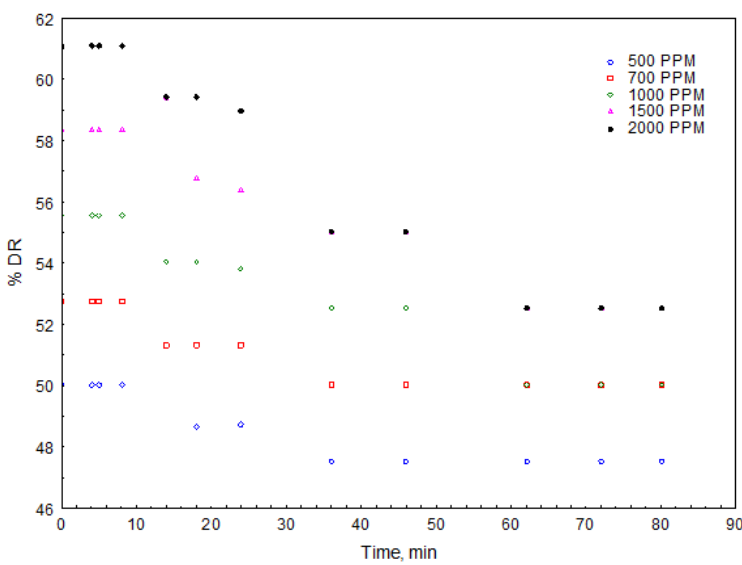

Fig. 7: The Effect of Time on Percent Drag Reduction for Different Polymer-Surfactant Concentrations at 3000 Rpm

The polymer - surfactant aggregate begins to influence flow individually, by increasing in Reynold's number this aggregate disintegrates and the surfactant micelles lose orientation, causing decreasing in percent drag reduction. The aggregate structure of polymer-surfactant can show stronger drag reduction than the substances alone with slower degradation and display drag reduction for a larger range of Reynolds numbers, this is clearly shown in Fig.7. This stability induced to that polymer enhances micelle stability by reducing the surface tension between the micelles hydrophobic cores and water. In order to get a direct image on the micelle and aggregate structures a cryo-transmission electron microscopy (cryo-TEM) techniques have been used in this research.

Figures (8-10) show the evolution of nanostructures and microstructures details of the inner structure of the aggregates for $(1000,1500$ and 2000) $\mathrm{ppm}$ respectively. The aggregate is formed by the surrounding of polymer chain (small disc) to the threadlike micelles of the surfactant (large disc) as shown in Fig.8.

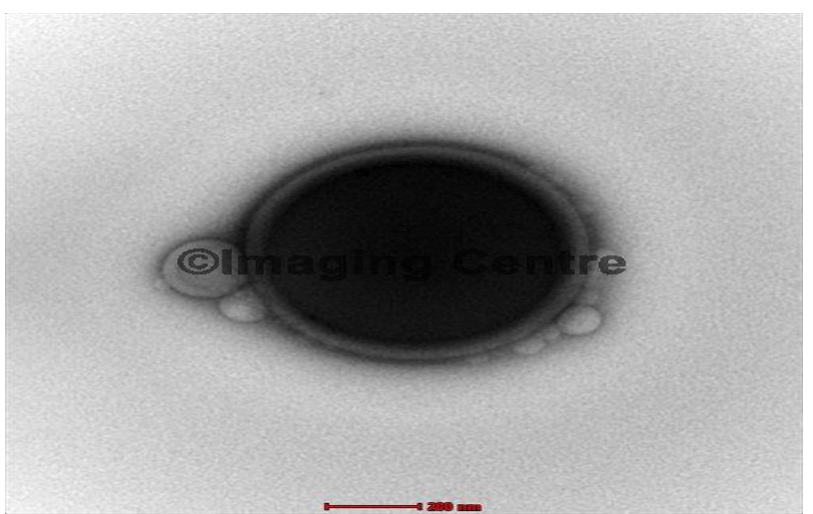

Fig. 8: CRYO-TEM Images of Polymer-Surfactant Additives at 1500 ppm Concentration 


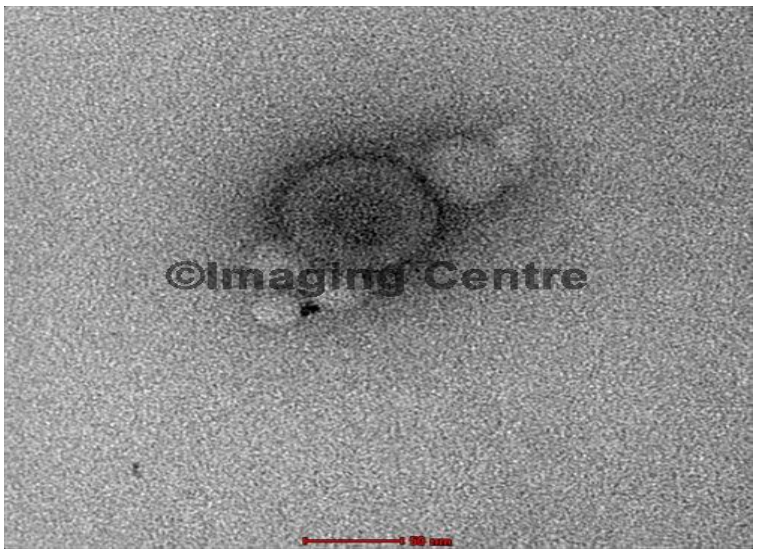

Fig. 9: CRYO-TEM Images of Polymer-Surfactant Additives at 1500 ppm Concentration

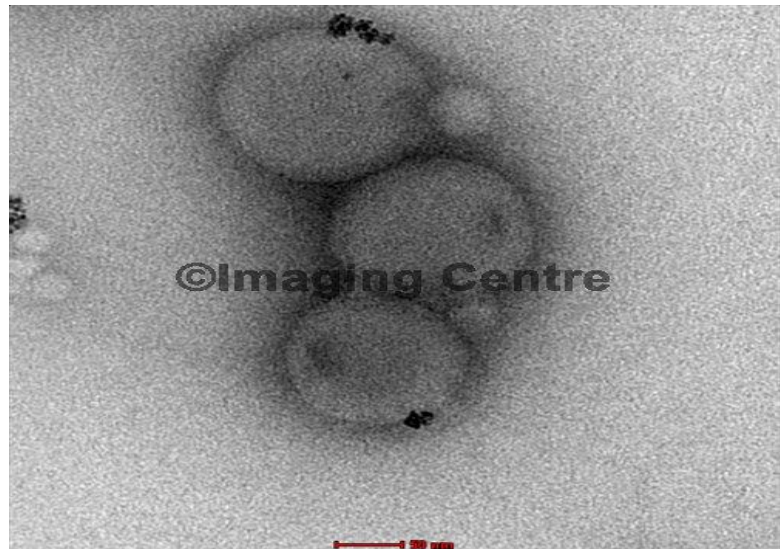

Fig. 10: CRYO-TEM Images of Polymer-Surfactant Additives at 2000 ppm Concentration

The CRYO-TEM images single particles, hence it's difficult to get the whole image of the population distribution of the aggregate, especially that TEM gives an image of the upper and lower part of the complexes superposed on each other and the image dependent on the relative position of the object to electron beam of the camera [17]. Thus in order to get a whole image on the aggregate structure depends on figs (8-10) and the microstructure of the surfactant and polymer. Weconcluded that the polymer decorates these cylindrical micelles as a hexagonal crystalline phase and the figures above are the cross-section of this hexagonal crystalline. A large and more aggregates forms with increasing polymer and surfactant concentration (see Fig.9) causing higher values of percent drag reduction as described [17-18].

\section{Conclusions}

It could be concluded from this work that the using a mix additives of polymer, and surfactant resulted in higher drag reduction than that using polymer or surfactant alone. The drag reduction increases with increasing additives concentration. Indeed there is no much difference in drag reduction values in using polymer or surfactant alone. The drag reduction of the mix additives shows more stable form among degradation due to the complexity of the aggregate structure that formed from the interaction between polymer and surfactant. Thecryo-transmission electron microscopy (cryo-TEM) techniques test shows the formation of this structure through arranging of polymer chain around surfactant micelles. It was suggested that the polymer chain decorated along the cylindrical micelles of the surfactant forming a hexagonal crystalline shape.

\section{References}

[1] Hassan, Y. and J. Ortiz-Villafuerte. 2002. Experimental study of micro-bubble drag reduction using particle image velocimetry. 11th International Symposium on Applications of Laser Techniques to Fluid Mechanics.

[2] Bewersdorff, H., 1986. Heterogeneous Drag Reduction in Turbulent Pipe Flow. In: B. GAMPERT, Ed, Springer Berlin Heidelberg, $337-348$.

[3] Azaiez, J., 2000. Reduction of free shear flows instability: effects of polymer versus fibre additives. Journal of Non-Newtonian Fluid Mechanics, 91(2): p. 233-254. http://dx.doi.org/10.1016/S0377-0257(99)00106-8

[4] Deutsch, S., et al., 2004. Microbubble drag reduction in rough walled turbulent boundary layers with comparison against polymer drag reduction. Experiments in fluids, 37(5): 731-744. http://dx.doi.org/10.1007/s00348-004-0863-6. 
[5] Ioannou, K. and N.O.A. Jorgen, P., 2005. Phase inversion in dispersed liquid-liquid flows. Exp. Therm. Fluid Sci. 29: 331-339. http://dx.doi.org/10.1016/j.expthermflusci.2004.05.003.

[6] Matras, Z., T. Malcher, and B. Gzyl-Malcher, 2008. The influence of polymer-surfactant aggregates on drag reduction. Thin Solid Films, 516(24):8848-8851. http://dx.doi.org/10.1016/j.tsf.2007.11.057.

[7] Segawa, T., et al., 2007. Turbulent drag reduction by means of alternating suction and blowing jets. Fluid dynamics research, 39(7):552-568. http://dx.doi.org/10.1016/j.fluiddyn.2007.01.002.

[8] Zhang, D.-Y., et al., 2011. Numerical simulation and experimental study of drag-reducing surface of a real shark skin. Journal of Hydrodynamics, Ser. B, 23(2):204-211.

[9] Zhen, L. and Y.A. Hassan, 2006. Wavelet autocorrelation identification of the turbulent flow multi-scales for drag reduction process in microbubbly flows. Chemical engineering science, 61(21):7107-7114. http://dx.doi.org/10.1016/j.ces.2006.07.031.

[10] Kazi, M.S.N., G.G. Duffy, and X.D. Chen, 1999. Heat transfer in the drag reducing regime of wood pulp fibre suspensions. Chemical Engineering Journal, 73(3):247-253. http://dx.doi.org/10.1016/S1385-8947(99)00047-9.

[11] Metzner, A. and A. Metzner, 1970. Stress levels in rapid extensional flows of polymeric fluids. Rheologica Acta, 9(2):174-181. http://dx.doi.org/10.1007/BF01973476.

[12] Deutsch, S., et al., 2006. Combined polymer and microbubble drag reduction on a large flat plate. Journal of Fluid Mechanics, 556: $309-327$. http://dx.doi.org/10.1017/S0022112006009487.

[13] Shenoy, A., 1984. A review on drag reduction with special reference to micellar systems. Colloid and Polymer Science, 262(4):319-337. http://dx.doi.org/10.1007/BF01410471.

[14] Missel, P.J., et al., 1980. Thermodynamic analysis of the growth of sodium dodecyl sulfate micelles. The Journal of Physical Chemistry, 84(9): 1044-1057. http://dx.doi.org/10.1021/j100446a021.

[15] Missel, P.J., et al., 1983. Influence of chain length on the sphere-to-rod transition in alkyl sulfate micelles. The Journal of Physical Chemistry, 87(7): 1264-1277. http://dx.doi.org/10.1021/j100230a032.

[16] Elson, T. and J. Garside, 1983. Drag reduction in aqueous cationic soap solutions. Journal of Non-Newtonian Fluid Mechanics, 12(2): 121133. http://dx.doi.org/10.1016/0377-0257(83)80033-0.

[17] Smith, R. and W. Tiederman, 1991. The mechanism of polymer thread drag reduction. Rheologica acta, 30(2): 103-113. http://dx.doi.org/10.1007/BF01134599.

[18] Nizri, G., et al., 2014. Microstructural characterization of micro-and nanoparticles formed by polymer-surfactant interactions. Langmuir, 20(11): 4380-4385. http://dx.doi.org/10.1021/la0364441.

[19] Grüneberger, R. and W. Hage, 2011. Drag characteristics of longitudinal and transverse riblets at low dimensionless spacings. Experiments in Fluids, 50(2): 363-373. http://dx.doi.org/10.1007/s00348-010-0936-7. 